Case report

\title{
Incomplete, atypical kawasaki disease or evolving systemic juvenile idiopathic arthritis: a case report Shakeel Shaikh ${ }^{1}$, Sidra Ishaque ${ }^{2 *}$ and Taimur Saleem ${ }^{2}$
}

\author{
Addresses: ${ }^{1}$ Department of Pediatrics and Child Health, The Aga Khan University, (Stadium Road), Karachi, 74800, Pakistan \\ ${ }^{2}$ Medical College, The Aga Khan University, (Stadium Road), Karachi, 74800, Pakistan \\ Email: SS - shakeel.shaikh@aku.edu; SI* - sidraishaque@gmail.com; TS - taimur@gmail.com \\ * Corresponding author
}

Received: 28 March 2009 Accepted: 16 July 2009 Published: 6 August 2009

Cases Journal 2009, 2:6962 doi: 10.4076/1757-1626-2-6962

This article is available from: http://casesjournal.com/casesjournal/article/view/6962

(C) 2009 Shaikh et al.; licensee Cases Network Ltd.

This is an Open Access article distributed under the terms of the Creative Commons Attribution License (http://creativecommons.org/licenses/by/3.0), which permits unrestricted use, distribution, and reproduction in any medium, provided the original work is properly cited.

\begin{abstract}
Kawasaki disease is an acute febrile condition seen in children. However, it is also well recognized that some patients do not fulfill the classic diagnostic criteria for the diagnosis of kawasaki disease. The incomplete form of kawasaki disease is termed as 'Incomplete KD' or 'Atypical KD'. We present a case of a 6 year old child with a history of prolonged fever, periorbital, oral and lip changes, changes in the extremities and an erythamatous, maculopapular rash. Based on the physical exam and her echocardiogram that showed right coronary artery dilatation, Intravenous immune globulin was administered in this patient. This patient was refractory to two doses of intravenous immune globulin and therefore was started on methylprednisolone, to which she responded dramatically. The diagnostic dilemma primarily arose when this child presented with joint pain a day after her discharge from the hospital and a positive laboratory workup. So, was this a case of incomplete Kawasaki refractory to intravenous immuno globulin therapy or systemic juvenile idiopathic arthritis? We suggest that physicians should be cognizant of the fact that they must individualize every patient's management to the best of their knowledge and judgment, rather than merely going by the guidelines.
\end{abstract}

\section{Case presentation}

A 6 years and 4 months old girl from Karachi, Pakistan presented with a 15 days history of fever, sore throat, dry and cracked lips, rash and peri-orbital swelling. The fever was high grade, intermittent and associated with chills and rigors. The rash was erythematous and patchy in distribution with involvement of the face and limbs. There was swelling on the body which was initially peri-orbital and then became more generalized to involve the extremities. She also had a history of reduced oral intake since the past ten days. Associated complaints included arthralgia without ostensible arthritis, diarrhea and several episodes of vomiting. Before coming to our hospital, she had been treated with amoxicillin, lincomycin, cefixime and clarithromycin for a week by a local physician without any improvement in signs or symptoms. She had received all her vaccinations as per EPI (extended program for immunization) schedule of Pakistan.

On examination, she had an irritable and toxic look with bilateral peri-orbital swelling and cracked lips. A strawberry tongue was seen on examination of the buccal cavity. 
An erythematous, maculopapular rash on the face and limbs was observed. Edema and induration of the limbs was also appreciated. No evidence of cervical lymphadenopathy or conjunctival injection was noticed (Figure 1). She was tachcardiac with a pulse of 160 beats per minute, febrile with a temperature of $39^{\circ} \mathrm{C}$. Her blood pressure was 105/66 mmHg.

With this history and presentation, the initial impression was of cellulitis, an acute hypersensitivity reaction or an incomplete kawasaki disease. She was admitted for observation and administered intravenous fluids and antibiotics (ceftriaxone and cloxacillin). Her baseline laboratory work up was sent. Abnormal laboratory findings included a low hemoglobin (9.3), a low hematocrit $(28 \%)$, raised white cell count $\left(38.4 \times 10^{9} / \mathrm{L}\right)$, with a predominance of neutrophils $(83.4 \%)$, and thrombocytosis (platelet count of 925). C-reactive protein and Erythrocyte Sedimentation Rate were raised $(24.4 \mathrm{mg} / \mathrm{dl}$ and $100 \mathrm{~mm} / \mathrm{hr}$ respectively). Albumin was lower than the normal range of $1.6 \mathrm{mg} / \mathrm{dl}$. The rest of the work-up including electrolyte and renal function workup was within the normal range.

In addition, her blood cultures showed no growth, her urine detailed report was normal and her liver function tests showed no abnormalities.

An echocardiogram was performed which showed a small patent foramen ovale (PFO), a normal left coronary artery

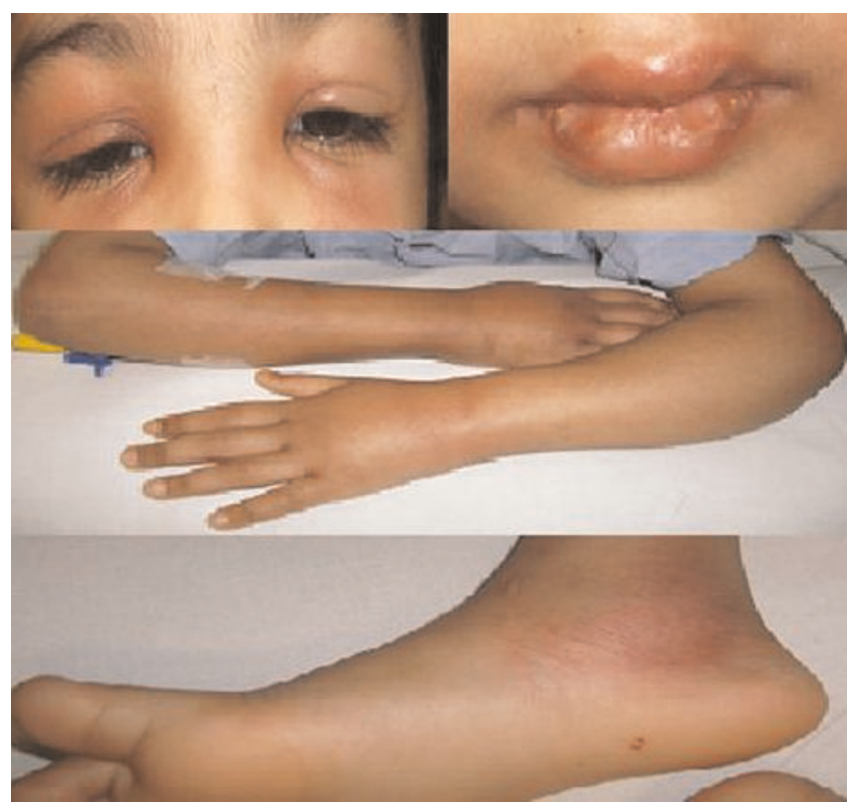

Figure I. 6 years old girl with peri-orbital erythema and swelling, cracked lips, swelling and rash of extremities. and right coronary artery dilatation with thrombus formation; with ostium measurements of $5.7-6.2 \mathrm{~mm}$ and measurements in the remaining artery between 3.2 $4 \mathrm{~mm}$ (Figure 2).

Based on the constellation of these findings, a diagnosis of incomplete kawasaki disease was made. She was given intravenous immunoglobulins (IVIG - $2 \mathrm{gm} / \mathrm{kg}$ ), aspirin (100 mg/kg/day) and acetaminophen as needed. However, the response to this management was sub-optimal. She continued to have persistent fever spikes; even after $>36$ hours of the completion of the first dose of IVIG and her inflammatory markers remained elevated with a C-reactive protein of $25 \mathrm{mg} / \mathrm{dl}$ and platelet count of $1365 \times 10^{9} / \mathrm{L}$. She was then given a second dose of IVIG. Her autoimmune profile was also sent at this stage which was negative (ANA, ASMA, AMA). She was given pulse therapy with intravenous methylprednisolone for three days after her failure to respond to the second dose of IVIG. In addition, she received clopidogerel, aspirin, ceftriaxone and vancomycin. She showed significant clinical improvement after institution of steroid therapy with resolution of fever spikes and normalization of inflammatory markers $(\mathrm{ESR}=55, \mathrm{CRP}=3.7$ and platelet $\left.=660 \times 10^{9} / \mathrm{L}\right)$. Repeat echocardiography showed no change as compared to previous study.

During the hospital stay, she developed the complaint of generalized abdominal pain. An ultrasound of the abdomen was performed which showed mild hepatomegaly with some echogenic areas in the renal parenchyma in both kidneys. This most likely represented protein casts. She was discharged from the hospital on the $13^{\text {th }}$ day of

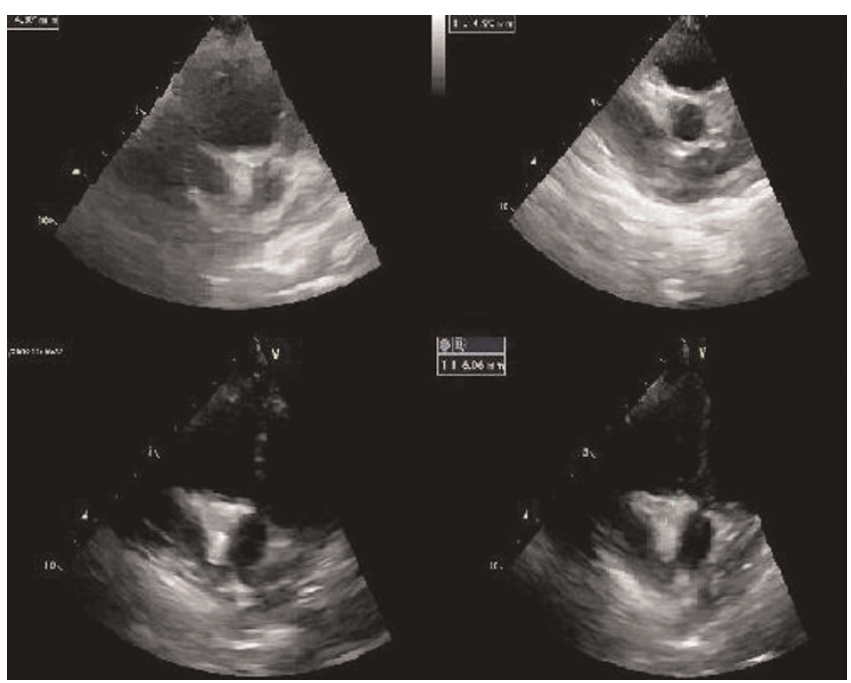

Figure 2. Echocardiographic images showing right coronary artery dilatation. 
admission on proton pump inhibitors, clopidogerel and aspirin.

However, one day after her discharge from the hospital, she returned to the emergency room with complaints of high grade fever and backache for one day. On examination, she had arthritis of the right knee joint and right hip joint. No abnormalities of the palms, soles and oral mucosa were noted and no rash or desquamation was seen. She was admitted and started on intravenous fluids, antibiotics and NSAIDs for relief of arthritis. Anti-nuclear antibody and Rheumatoid Factor were also sent which came back to be borderline high. An aortogram was also done to better visualize the coronary arteries; dilatation of the right coronary artery was not seen on this study. A diagnosis of evolving Systemic Juvenile Idiopathic Arthritis was made because of the short time duration $(<6$ weeks). She was started on steroids, proton pump inhibitor, naproxen and clopidogerel and discharged after an uneventful stay of 6 days. Her follow-up in the clinic after a week showed improvement with a CRP of 0.3 $\mathrm{mg} / \mathrm{dl}$, platelets of $668 \times 10^{9} / \mathrm{L}$, ESR $=5 \mathrm{~mm} / \mathrm{hr}$ and Hemoglobin of $13.6 \mathrm{~g} / \mathrm{dl}$ ).

She has been improving clinically over the last few months. All her recent follow ups have been unremarkable in terms of any fever, joint pain, swelling or limitation of activity. The child is healthy, active and playful with no active complaints. Her systemic exams have also been unremarkable so far. Her last EDSR and CRP were within the normal range. She has been off steroids since the last month. She was started on 4 tablets of prednisone, which has been tapered down to zero over the last five months. Her current therapy comprises of Tab Naproxen Sodium (once daily).

\section{Discussion}

Kawasaki disease (KD) is an acute febrile condition seen in children. Even though it was first reported in Japan about 30 years ago, the original diagnostic criteria defined by Dr Tomisaku Kawasaki in 1967 are still authentic and widely used today. However, it is also well recognized that some patients do not fulfill the classic diagnostic criteria for the diagnosis of $\mathrm{KD}$. The incomplete form of $\mathrm{KD}$ is termed as 'Incomplete $\mathrm{KD}^{\prime}$ or 'Atypical KD'. Because incomplete $\mathrm{KD}$ is not a mild form of $\mathrm{KD}$, children remain at similar risk for cardiovascular sequelae as that of complete KD [1]. Since the disease has a similar risk of coronary artery abnormalities (CAA) as complete KD [2-5], it is necessary to make an accurate diagnosis in order to prevent the development of coronary artery abnormalities CAA [1-4]. "Incomplete" KD is the preferred term, as these patients do not appear to differ from those with classic KD in any way except that they lack a sufficient number of criteria to fulfill the epidemiologic case definition [5]. The American Heart Association guidelines have been used for the diagnosis of incomplete $\mathrm{KD}$, which is based on echocardiographic findings and laboratory findings [6].

Cervical lymphadenopathy is the cardinal manifestation most often absent in children with incomplete KD as was observed in our patient [1], this is followed by rash, peripheral extremity and mucous membrane changes [7]. Because of the prolonged unexplained history of fever, the typical age group of the patient and the rash, incomplete KD was considered in our patient.

The guidelines for the evaluation and treatment of patients in whom incomplete $\mathrm{KD}$ is suspected were established by the American Heart Association in 2004. [6] Our patient had a 15 day history of fever and three of the principal clinical criteria. Clinical signs and symptoms were consistent with $\mathrm{KD}$. According to the algorithm of the guidelines, assessment using laboratory tests was required [1]. The tests showed that the patient had a C-reactive protein level of $\geq 3.0 \mathrm{mg} / \mathrm{dL}$ and fulfilled $\geq 3$ supplemental laboratory criteria (albumin $\leq 3.0 \mathrm{~g} / \mathrm{dL}$, anaemia for age, platelets after 7 days $\geq 45 \times 104 / \mu \mathrm{L}$, WBC counts $\geq 15000 /$ $\mu \mathrm{L}$ and urine $\geq 10 \mathrm{WBCs} /$ high-power field). Therefore, we considered this patient to be suffering from incomplete KD.

She was administered the first dose of IVIG, keeping in mind her clinical presentation and laboratory parameters. Data supports the use of IVIG while there is ongoing inflammation (usually taken as ongoing fever or raised acute inflammatory markers. One high quality systemic review of sixteen RCTs showed that IVIG is of benefit in treating kawasaki disease. [19]. In children with fever and classic clinical and laboratory findings of $\mathrm{KD}$, treatment with IVIG resulted in better coronary outcomes and decreased the total length of time of clinical symptoms [20].

Immunosuppressive therapy was administered in this case to control the symptoms after two doses of refractory IVIG treatment. There could be two considerations in the light of the stated scenario. It could have been a case of incomplete $\mathrm{KD}$ that was refractory to IVIG therapy, responding later to methyl prednisolone. Failure to respond usually is defined as persistent or recrudenscent fever more than 36 hours after completion of the initial IVIG infusion [8]. The infusion of intravenous immunoglobulin (IVIG) within 10 days of the onset of KD is known to reduce both the duration of fever and the incidence of coronary artery disease, and thus together with aspirin are the standard treatment $[9,10]$. However, approximately $15-20 \%$ of the children with KD have a persistent or recurrent fever and a progression of coronary 
dilatation despite IVIG treatment $[8,11,12]$. Studies support the use of corticosteroids in most patients with IVIGrefractory $\mathrm{KD}[13,14]$. Wright et al first described the outcome in patients with IVIG-resistant KD who were treated with pulsed doses of corticosteroid. All the patients were treated successfully with pulse methylprednisolone with no adverse effects noted. A recent review article suggests that pulse methylprednisolone therapy should be considered if there is no response to two standard doses of IVIG treatment [15].

The other possibility is that this was a case of an evolving systemic juvenile idiopathic arthritis (SJIA), given the less than 6 week presentation, with fever, arthritis, rash and a positive lab workup. The main dilemma is that neither KD nor SJIA have absolutely specific diagnostic laboratory tests. Both diseases show similar laboratory findings with elevated WBC counts, elevated C-reactive protein, leukocytosis, thrombocytosis and anaemia and these account for diagnostic confusion. Moreover, the initial presentation of SJIA appears similar to incomplete KD. Prolonged fever and erythematous rash are common findings in both incomplete KD and SJIA [16]. These findings suggest that SJIA cannot be easily distinguished from incomplete KD. In our case, the clinical and diagnostic dilemma primarily arose when the patient presented with joint pain a day after her discharge from the hospital and a positive laboratory workup [16].

The echocardiogram plays a key role in making a diagnosis of incomplete $\mathrm{KD}$. However, aneurysms rarely form before the 10th day of illness $[17,18]$. However, instituting IVIG therapy within the first 10 days of illness is recommended [16].

Therefore, physicians are always in a dilemma over whether to first make an accurate diagnosis or begin early treatment to prevent the development of CAA. IVIG therapy is not only a costly intervention but it also exposes the patients to unnecessary risks related to receiving a blood product [18], the diagnosis of incomplete $\mathrm{KD}$ requires sufficient clinical evidence. Although an aortogram done later in our patient showed no abnormal findings, her clinical features, laboratory findings and echocardiographic findings were sufficiently strong evidence to warrant a working diagnosis of incomplete $\mathrm{KD}$ and therefore IVIG was administered.

It is important to recognize that as long as the diagnosis of $\mathrm{KD}$ is based upon clinical criteria, it will remain a deficient undertaking. As long as the disadvantages of undertreating appear to outweigh the disadvantages of overtreating, IVIG will be used injudiciously to treat these children. However, this approach of treating children at risk even though the diagnosis is uncertain should result in fewer children with incomplete KD going untreated and subsequently developing coronary artery aneurysms. Hopefully, with increasing knowledge, diagnostic accuracy will improve over time, thereby ensuring that proper therapy is instituted correctly in patients with KD and SJIA.

\section{Conclusion}

We have presented here a case causing considerable diagnostic dilemma. Physicians need to recognize that systemic-onset juvenile idiopathic arthritis can be mistaken for incomplete kawasaki disease, despite the use of established guidelines.

\section{Abbreviations}

AMA, Anti mitochondrial antibody; ANA, Anti nuclear antibody; ASMA, Anti Smith antibody; CAA, Coronary artery aneurism; CRP, C- reactive protein; ESR, Erythrocyte sedimentation rate; IVIG, Intravenous immune globulin; KD, kawasaki disease; NSAIDS, Non steroidal anti inflammatory drugs; SJIA, Systemic juvenile idiopathic arthritis.

\section{Consent}

Written informed consent was obtained from the patient for publication of this case report and accompanying images. A copy of the written consent is available for review by the Editor-in-Chief of this journal.

\section{Competing interests}

The authors declare that they have no competing interests.

\section{Authors' contributions}

SS was involved in interpretation of the patient data and providing overall supervision in the project. SI conceived the idea of the manuscript, and was a major contributor in data collection and writing the manuscript. TS participated in the data collection and sequencing and drafting of the manuscript. All authors read and approved the final manuscript.

\section{References}

I. Royle JA, Williams K, Elliott E, Sholler G, Nolan T, Allen R, Isaacs D: Kawasaki disease in Australia, 1993-1995. Arch Dis Child 1998, 78:33-39.

2. Rowley AH, Gonzalez-Crussi F, Gidding SS, Duffy CE, Shulman ST: Incomplete kawasaki disease with coronary artery involvement. J Pediatr 1987, I I 0:409-4I3.

3. Witt MT, Minich LL, Bohnsack JF, Young PC: Kawasaki disease: more patients are being diagnosed who do not meet American Heart Association criteria. Pediatrics 1999, 104:el0.

4. Stapp J, Marshall GS: Fulfillment of diagnostic criteria in kawasaki disease. South Med J 2000, 93:44-47.

5. Rowley $\mathrm{AH}$ : Incomplete (atypical) kawasaki disease. Pediatr Infect Dis J 2002, 21:563-565.

6. Newburger JW, Takahashi M, Gerber MA, Gewitz MH, Tani LY, Burns JC, Shulman ST, Bolger AF, Ferrieri P, Baltimore RS, Wilson WR, Baddour LM, Levison ME, Pallasch TJ, Falace DA, Taubert KA: Diagnosis, treatment, and long-term management of kawasaki disease. a statement for health professionals from the Committee on Rheumatic Fever, 
Endocarditis, and kawasaki disease, Council on Cardiovascular Disease in the Young, American Heart Association. Circulation 2004, I I 0:2747-27I7.

7. Fukushige J, Takahashi N, Ueda Y, Ueda K: Incidence and clinical features of incomplete kawasaki disease. Acta Paediatr 1994, 83:1057-1060.

8. Kakish KS, Dhaheri ASW, Othman SB: Intravenous Immunoglobulin - Resistant kawasaki disease Treated with Pulsed Doses of Methylprednisolone. Bahrain Med Bull 2008, 30:I-5.

9. Furusho $K$, Kamiya $T$, Nakano $H$, Kiyosawa $N$, Shinomiya $K$, Hayashidera T, Tamura T, Hirose O, Manabe Y, Yokoyama T et al.: High dose intravenous Gamma-globulin for kawasaki disease. Lancet 1984, 10:1055-1058.

10. Newburger JW, Takahashi M, Burns JC, Beiser AS, Chung KJ, Duffy CE, Glode MP, Mason WH, Reddy V, Sanders SP et al.: The Treatment of Kawasaki Syndrome with Intravenous Gamma globulin. N Engl J Med 1986, 315:34|-347.

II. Ashouri N, Takahashi M, Dorey F, Mason W: Risk factors for nonresponse to therapy in kawasaki disease. J Pediatr 2008, 153:365-368.

12. Newburger JW, Takahashi M, Beiser AS, Burns JC, Bastian J, Chung KJ, Colan SD, Duffy CE, Fulton DR, Glode MP et al.: A Single Intravenous Infusion of Gamma globulin as Compared with Four Infusions in the Treatment of Acute Kawasaki Syndrome. N Engl J Med 1991, 324:1633-1639.

13. Lang BA, Yeung RS, Oen KG, Malleson PN, Huber AM, Riley M, Ebbeson R, Ramsey SE, Laxer RM, Silverman ED, McCrindle BW, Ratnapalan S, Feldman BM: Corticosteroid treatment of refractory kawasaki disease. J Rheumatol 2006, 33:803-809.

14. Raman V, Kim J, Sharkey A, Chatila T: Response of refractory kawasaki disease to pulse steroid and cyclosporin A therapy. Pediatr Infect Dis / 200I, 20:635-637.

15. Hung JJ, Chiu CH: Pulse methylprednisolone therapy in the treatment of immune globulin-resistant kawasaki disease: case report and review of the literature. Ann Trop Paediatr 2004, 24:89-93

16. Komatsu $\mathrm{H}$, Akihiko Tateno A: Failure to distinguish systemiconset juvenile idiopathic arthritis from incomplete kawasaki disease. J Paediatr Child Health 2007, 43:707-709.

17. Gupta-Malhotra M, Rao PS: Current perspectives on kawasaki disease. Indian J Pediatr 2005, 72:62I-629.

18. Burns JC, Capparelli EV, Brown JA, Newburger JW, Glode MP: Intravenous Gamma-globulin Treatment and Retreatment in kawasaki disease. Pediatr Infect Dis J 1998, I7: I I44-I I 48.

19. Criteria for the clinical use of intravenous immunoglobulin in Australia, 2007 [http://www.nba.gov.au/ivig/pdf/criteria.pdf].

20. Tse SM, Silverman ED, McCrindle BW, Yeung RS: Early treatment with intravenous immunoglobulin in patients with kawasaki disease. J Pediatr 2002, 140:450-455.

\section{Do you have a case to share?}

\section{Submit your case report today}

- Rapid peer review

- Fast publication

- PubMed indexing

- Inclusion in Cases Database

\section{Any patient, any case, can teach us something}

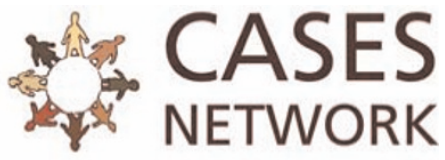

www.casesnetwork.com 